\title{
Size Dependence of the Magnetic Relaxation and Specific Power Absorption in Iron Oxide Nanoparticles
}

\author{
E. Lima Jr. - T. E. Torres - L. M. Rossi - H. \\ R. Rechenberg - T.S. Berquo - A. Ibarra * C. \\ Marquina · R. M. Ibarra - G. F. Goya
}

Received: date / Accepted: date

\begin{abstract}
We report on the magnetic and power absorption properties of a series of iron oxide nanoparticles with average sizes $<\mathrm{d}>$ ranging from 3 to $23 \mathrm{~nm}$, prepared by thermal decomposition of Iron(III) acetylacetonate in organic media. From the careful characterization of the magnetic and physicochemical properties of these samples, we were able to reproduce the specific power absorption (SPA) values experimentally found, as well as their dependence with particle size, using a simple model of Brownian and Néel Relaxation at room temperature. SPA experiments in ac magnetic fields $\left(0_{0}=13 \mathrm{kA} / \mathrm{m}\right.$ and $\left.f=250 \mathrm{kHz}\right)$ indicated that the magnetic and rheological properties played the fundamental rule to determine the heating efficiency at different conditions. A maximum SPA value of $344 \mathrm{~W} / \mathrm{g}$

E. Lima Jr.

CONICET \& Instituto de Nanociencia y Nanotecnologia \& Centro Atómico Bariloche, S. C. Bariloche, Argentina

T. E. Torres

Instituto de Nanociencia de Aragón (INA) \& Departamento de Física de la Materia Condensada \& Laboratorio de Microscopias Avanzadas (LMA), University of Zaragoza, Zaragoza, Spain

L. M. Rossi

Instituto de Química Universidade de São Paulo, São Paulo, Brazil

H. R. Rechenberg

Instituto de Física - Universidade de São Paulo, São Paulo, Brazil

T. S. Berquo

Institute of Rock Magnetism, University of Minnesota, Minneapolis, USA

A. Ibarra

INA \& LMA, University of Zaragoza, Zaragoza, Spain

C. Marquina

Departamento de Física de la Materia Condensada \& Instituto de Ciencia de Materiales de Aragón (ICMA), CSIC-Universidad de Zaragoza, Zaragoza, Spain.

R. M. Ibarra

INA \& Departamento de Física de la Materia Condensada \& LMA, University of Zaragoza, Zaragoza, Spain

G. F. Goya

INA \& Departamento de Física de la Materia Condensada, University of Zaragoza, Zaragoza, Spain Tel.: +34-876 555362

Fax: +123-976 762776

E-mail: goya@unizar.es
\end{abstract}


was obtained for a sample containing nanoparticles with $<\mathrm{d}>=12 \mathrm{~nm}$ and dispersion $\sigma=$ 0.25 . The observed SPA dependence with particle diameter and their magnetic parameters indicated that, for the size range and experimental conditions of $\mathrm{f}$ and $\mathrm{H}$ studied in this work, both Néel and Brown relaxation mechanisms are important to the heat generation observed.

Keywords Magnetic Nanoparticles · Magnetic Losses - Superparamagnetism · Electromagnetic Heating

\section{Introduction}

The ability to synthesize uniform nanoparticles is a central issue for many biomedical applications since the physical properties of the nanoparticles are strongly dependent on their dimensions (Kim et al (2011)). Recently, nanoparticle-based magnetic fluid hyperthermia (MFH) has emerged as a potential candidate for improving the classical hyperthermia therapy in oncology (Maier-Hauff et al (2011), Landeghem et al (2009), Johannsen et al (2010), Thiesen et al (2008)) known since centuries ago (Alexander (2003)). Within the Magnetic Hyperthermia (MH) strategy, the death of a target malignant tissue is achieved by the increment of the local temperature generated by the magnetic nanoparticles (MNPs) when exposed to a time-varying magnetic field. This application of MNPs as heating agents requires non-toxicity, colloidal stability and biodegradability (Goya et al (2008)). The most spread materials studied for $\mathrm{MFH}$ applications are magnetite $\left(\mathrm{Fe}_{3} \mathrm{O}_{4}\right)$ and its related ferric oxide maghemite $\left(\gamma-\mathrm{Fe}_{2} \mathrm{O}_{3}\right)$, since they are the only magnetic nanomaterials approved for human uses so far. To improve stability and biocompatibility the MNPs are coated with a polysaccharide and suspended in water-based solvents, but the effects of these coatings on the efficacy in Magnetic Resonance Imaging (MRI) or MFH applications is still under debate (Tanaka et al (2010)).

A key factor to increase the efficiency of nanoparticles in MFH clinical protocols is a high Specific Power Absorption (SPA) in a bio-friendly frequency range (radio-frequency). A high SPA of nanoparticles within a target tissue (a tumour, for example) allows a fine control over affected area, reducing the damage to the healthy tissue. Moreover, large SPA values imply shorter exposition times of the organism to the magnetic material used and to the radio-frequency magnetic field applied, as well as a better efficiency in the heating process of the target tissue. The highest values of SPA reported so far for iron-oxide nanoparticles was about $900 \mathrm{~W} / \mathrm{g}$ for magnetosomes (Alphandery et al (2011), Hergt et al (2005)). For synthetic iron-oxide NPs, Hergt et al (2005) have observed a SPA about $400 \mathrm{~W} / \mathrm{g}$ for MNPs in an applied field of $16 \mathrm{kA} / \mathrm{m}$ and $400 \mathrm{kHz}$, while Goya et al (2008) measured $\sim 150 \mathrm{~W} / \mathrm{g}$ for MNPs with bimodal size distribution and in an applied field with $13 \mathrm{kA} / \mathrm{m}$ and $250 \mathrm{kHz}$. The ability to absorb radiated power from an alternating magnetic field at low frequencies (i.e., $<10^{6} \mathrm{~Hz}$ ) depends on the magnetic losses (fluctuation of magnetization through energy barriers), which is determined by the magnetic properties of the system (anisotropy, volume of the particle and magnetization), and the rheological properties of the target physiological medium (i.e. cell culture or tissue), which determines the mechanical relaxation. Looking for the physical parameters that optimize the power absorption efficiency of a ensemble of nanoparticles, it is important to remark the competition of two main mechanisms: the mechanical mechanism that is the physical movement of the nanoparticles in the fluid (Brownian movement) (Goya et al (2007)), and the magnetic mechanism, which could be the Néel relaxation (for a SPM system) or hysteresis losses (for a blocked system) (Carrey et al (2011)). For single-domain superparamagnetic NPs in a colloidal suspension, the physical 
mechanisms involved are both Brownian and Néel magnetic relaxation, which cab assumed as parallel process according to Rosensweig (2002).

In a previous work (Goya et al (2008)), we have demonstrated that the high power absorption of monodomain magnetic nanoparticles in an ac magnetic field in radio-frequency range is not straight associated to the magnetization of saturation MS, with the high SPA apparently connected to the particle volume, the size distribution, and the effective MNPs anisotropy. Here, we undertake a systematic work on the power absorption of ferrite nanoparticles with average particle sizes $<\mathrm{d}>$ ranging from 7 to $25 \mathrm{~nm}$. Our results confirm that the heating generation in our superparamagnetic iron-oxide nanoparticles, as measured through the SPA $(\mathrm{H}=13 \mathrm{kA} / \mathrm{m}, \mathrm{f}=250 \mathrm{kHz})$, are originated from both Néel and Brown relaxations and consequently the size dependence of SPA can be explained using the simple model of Néel and Brown relaxation of the magnetic moment as proposed by Rosensweig (2002). By experimentally determining all the key parameters involved in this model,for each sample, we were able to reproduce with hogh precision the SPA values found in our iron-oxide MNPs.

\section{Materials and Methods}

The synthesis of the ferrite MNPs was based on the high-temperature decomposition of the precursor $\mathrm{Fe}(\mathrm{acac})_{3}$ in the presence of a long-chain alcohol (1,2 octanediol) and surfactants (oleic acid and oleilamine) as reported by Sun et al (2002) and Sun et al (2004), and using diphenyl ether (boiling point $\sim 533 \mathrm{~K}$ ) as organic medium. For NPs with $<\mathrm{d}><10 \mathrm{~nm}$, we have used a single-step procedure and the final particle sizes were tailored through the molar ratio $\left[\mathrm{Fe}(\mathrm{acac})_{3}\right] /[$ surfactants $]$ as described in Vargas et al (2005). The synthesis lasted for 120 minutes in argon flux $(\sim 0.5 \mathrm{~L} / \mathrm{min}$. $)$ in reflux regime at $\mathrm{T}=533 \mathrm{~K}$. Three samples with $<\mathrm{d}>=3,7$ and $9 \mathrm{~nm}$, labeled AP01, AP02 and AP03, respectively, were synthesized by this single-step procedure. NPs with $<\mathrm{d}>>10 \mathrm{~nm}$ were grown using previously synthesized seeds and the same protocol, in order to further increase the final particle size, similarly to that used by Torres et al (2010) for the system $\mathrm{CoFe}_{2} \mathrm{O}_{4}$. The set of samples grown initially from the $10 \mathrm{~nm}$ particles were labeled as GEYY, where YY indicates the number of times the re-crystallization took place (running from 01 to 03 ). The sequence goes like this: particles from sample AP03 $(9.5 \mathrm{~nm})$ were used as seeds for GE01 synthesis, GE01 as seeds of the GE02, and finally GE02 as seeds for GE03. For theses samples, we used $50 \mathrm{mg}$ of seeds and $2 \mathrm{mmol}$ of precursor, except for sample GE03, where we have used $20 \mathrm{mg}$ of seeds. Since the reaction time at high-temperature is incremented for each sample, the total crystallization time increases from $120 \mathrm{~min}$. (10 $\mathrm{nm}$ seeds) to $480 \mathrm{~min}$. for GE03. After the synthesis, ethanol was added to the solution and the particles were precipitated by centrifugation (7000 r pm/ 10min.). This washing and centrifugation procedure was repeated three times. Samples CYY were synthesized in a similar way, but using $3 \mathrm{~nm}$ NPs (sample AP01) as the first seed. In total, this set contains six samples: C01-C06. In order to reduce the particle size dispersion in samples CYY when compared with samples GEXX (see in Table 1), we increase the molar relation [seeds] $/[$ precursor] to $80 \mathrm{mg} / 2 \mathrm{mmol}$. In this case, the centrifugation protocol was set at $5000 \mathrm{rpm} / 40 \mathrm{~min}$. and two more steps of $15000 \mathrm{r} \mathrm{mm} / 10 \mathrm{~min}$. in order to only separate the larger particles selected in the first step. Transmission electron microscopy (TEM) and High-Resolution TEM (HRTEM) images were obtained in a transmission electron microscope (FEI TECNAI T20 and JEOL 2010F from FEI and Jeol company respectively), after dropping the colloidal solution onto a carboncoated copper grid. The ac susceptibility measurements with frequencies from 0.1 to $1 \mathrm{kHz}$ and field amplitude of $160 \mathrm{~A} / \mathrm{m}$, were performed as a function of temperature from 2 to 300 
$\mathrm{K}$ with a Quantum Design SQUID magnetometer, as well as the magnetization curves as function of temperature and applied field. The experimental SPA measurements were made in a home-made ac-field applicator, consisting of a resonant LC tank working at $\mathrm{f}=220-260$ $\mathrm{kHz}$, and field amplitudes from 0 to $12.7 \mathrm{kA} / \mathrm{m}$, and equipped with a quasi-adiabatic sample holder (losses by irradiation estimated in 15, see Natividad et al (2009)), working with 0.5 to $1 \mathrm{ml}$ of ferrofluid. Temperature data in this experiment were taken using a fiber optic temperature probe immune to radio-frequency environments. The experiments were carried at $1 \%$ wt. as standard concentration for all samples, with the NPs dispersed in Toluene.

\section{Experimental Results}

\subsection{Structural and Magnetic Characterization}

Analysis of particle size and morphology using bright-field TEM images (fig. 1) for samples APXX (single-step synthesis) showed the gradual increase of particle size with increasing molar precursor/surfactant ratio, with $<\mathrm{d}\rangle=3,7$ and $9 \mathrm{~nm}$ for samples AP01, AP02 and AP03, respectively. The size distributions of the APXX particles are well fitted with a Lognormal function and the morphology was found to be spherical-rounded.

Further increase of sizes could be achieved by re-growing the particles by the seed method described in the previous section, up to $25 \mathrm{~nm}$. For samples GE01-GE03, the size dispersion increased somewhat for each successive synthesis. These samples exhibited a bi-modal distributions, with maxima at $<\mathrm{d}>=11$ and $14 \mathrm{~nm}$ for samples GE01 and GE02, respectively, and a minority amount of small nanoparticles with particles size about $3 \mathrm{~nm}$ (see Goya et al (2008)). The diameter of the smaller particles observed correspond roughly to the size of the initial ferrite nucleus formed after the decomposition of the precursor and the amount of smaller particles changes with the re-crystallization time, suggesting that the contribution of smaller average sizes results from a fraction of seeds that remain without growing. The diameter histogram of sample GE03 present maxima at 17 and $26 \mathrm{~nm}$. Probably, the broad size dispersion with bimodal size distribution in these samples is related to the synthesis procedure, where we worked with a low concentration of seeds in the re-growth process.

TEM images of samples CYY are presented in fig. 2. The mean diameter of the nanoparticles did not continuously increases after each recrystallization step, as observed in the histograms presented in the respective insets. The values of mean diameter $d$ and the size distribution of samples CYY are obtained from the fitting of the diameter histogram with a lognormal function and the values obtained are given in Table 1, together with those obtained for samples APXX and GEXX. Comparing the size distribution of samples CYY and GEXX, we observe a narrower grain size distributions for samples C01-C06, indicating that the increment in the molar ratio seeds:precursor and the different centrifugation protocols were effective to reduce the dispersion.

HRTEM CYY nanoparticles confirms their high crystallinity of the particles in all samples and the First Fourier Transformation (FFT-HRTEM) confirms the spinel structure of the ferrites (see fig. 3 for samples $\mathrm{C} 01$ and $\mathrm{C} 02$; the insets show the respective diffraction patterns for the selected zone of the images, which can be indexed to crystalline planes of the spinel structure), which is confirmed by the XRD profiles (not shown). The crystalline characterization of samples APXX and GEXX and magnetic properties were presented elsewhere (Goya et al (2008)).

The magnetization loops of samples C01-C06 measured at $250 \mathrm{~K}$ are presented in fig. 4a. We observe the absence of the coercivity for all samples (superparamagnetic regime) as 

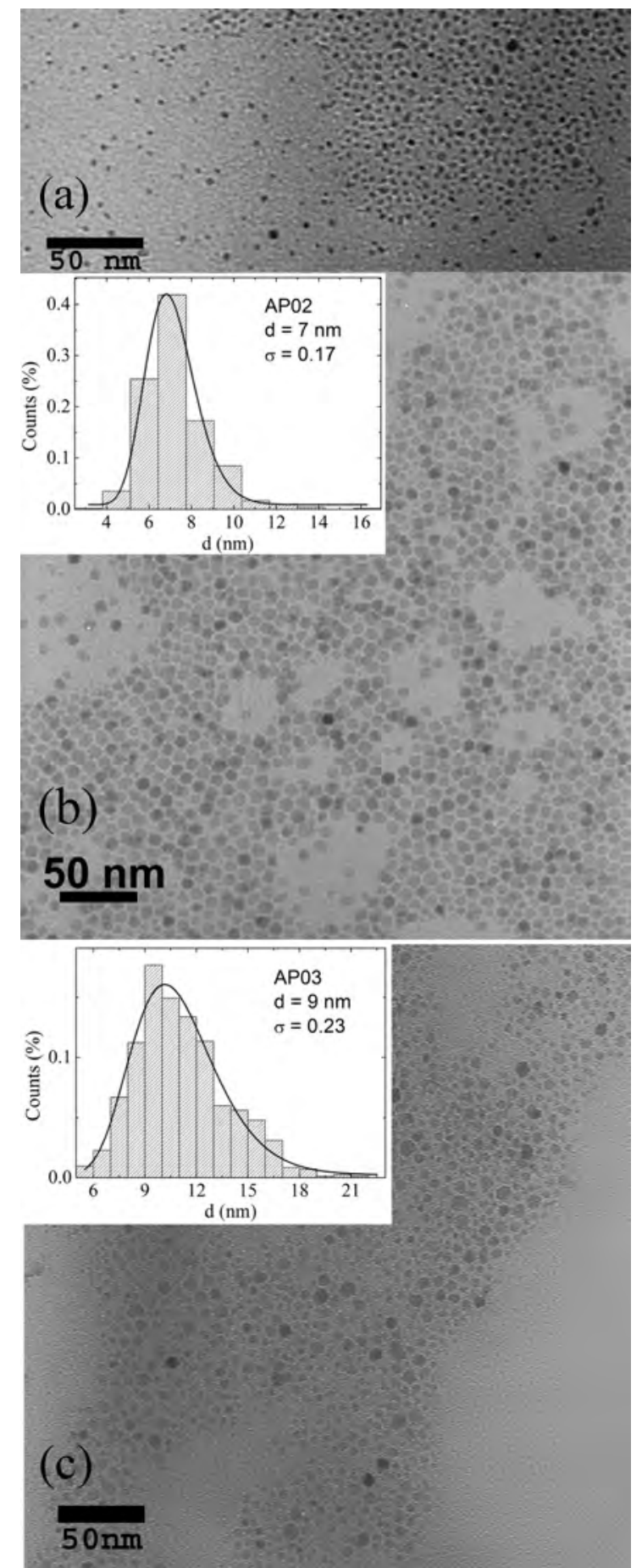

Fig. 1 TEM images of samples AP01 (a), AP2(b), and AP03 (c). Insets show the respective histograms fitted with a Lognormal distribution. 


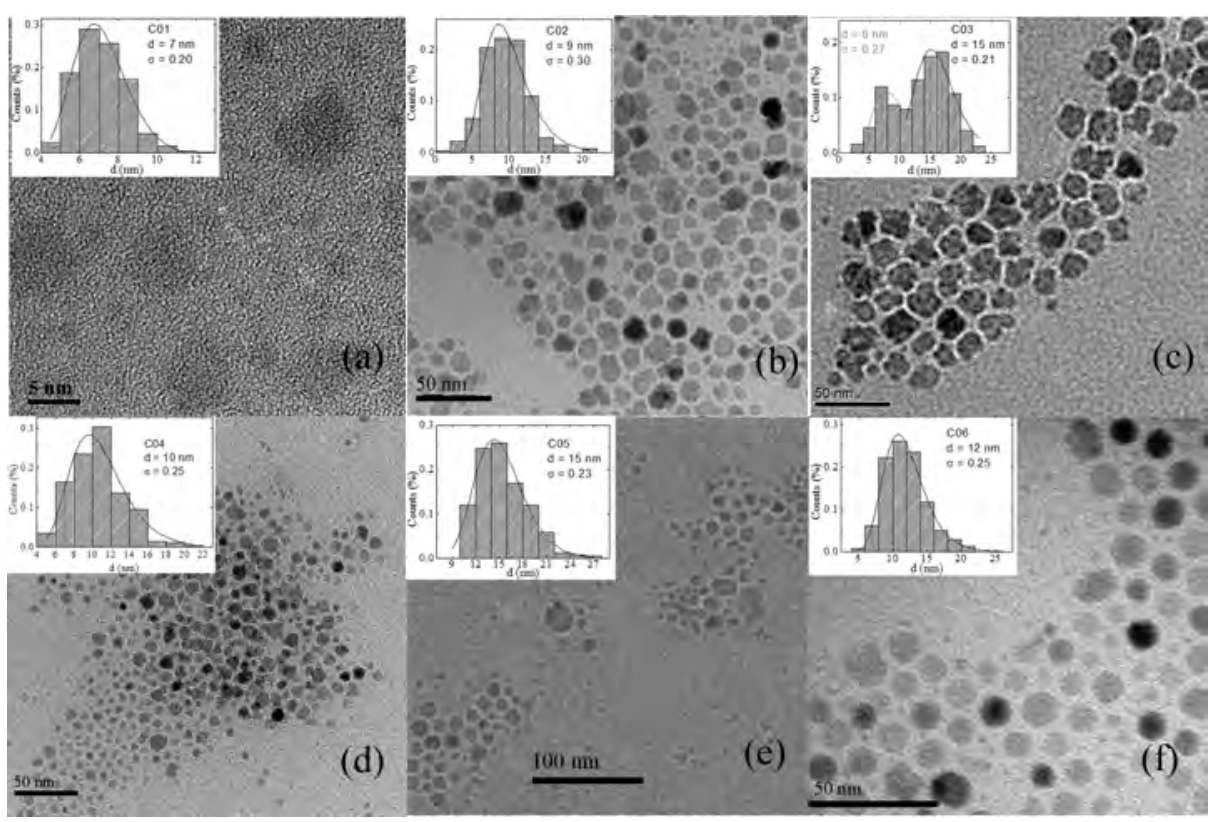

Fig. 2 TEM images of samples C01-C06 (a-f, respectively) with the corresponding histograms (insets) fitted with a Lognormal distribution (solid line).
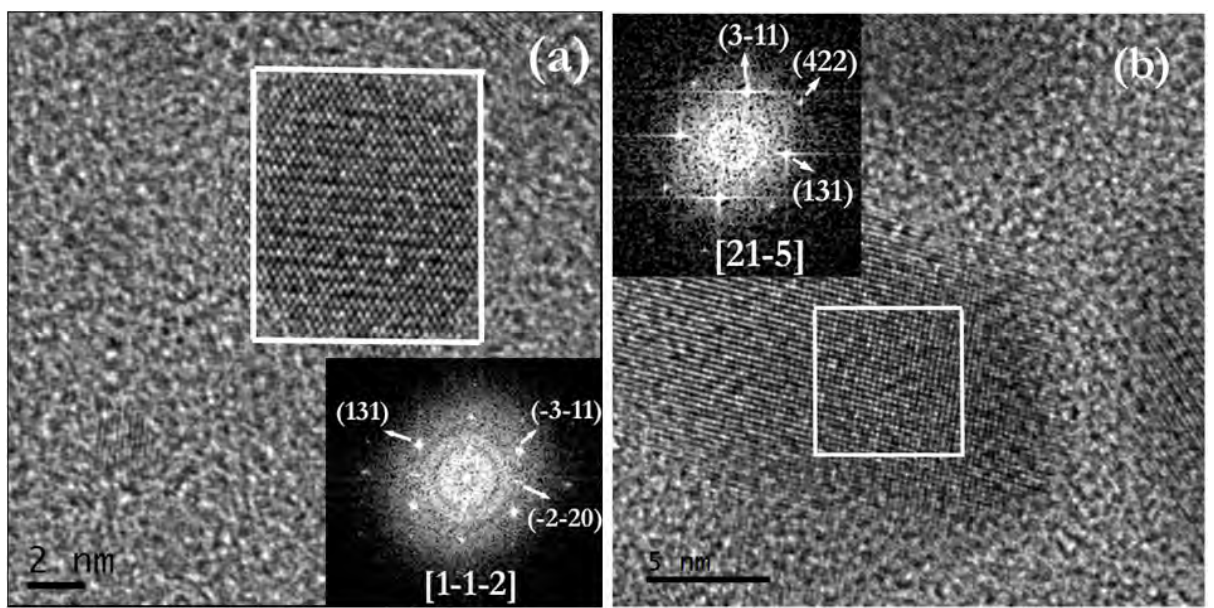

Fig. 3 HRTEM images of samples C01 (a) and C02 (b), The insets show the respective diffraction patterns for the selected zone of the images, which can be indexed to crystalline planes of the spinel structure.

expected from the ac measurement data showed below. The saturation magnetization $M_{S}$ at $250 K$ and at $5 K$ (see inset of fig. 4-a) decrease with decreasing the particle diameter. For larger particles, we observe highest MS values, being about $400 \mathrm{kA} / \mathrm{m}$ at $250 \mathrm{~K}$ for samples with $\mathrm{d}>8 \mathrm{~nm}$.

Magnetization measurements of samples $\mathrm{C} 01-\mathrm{C} 06$ as function of the temperature measured with $8 \mathrm{kA} / \mathrm{m}$ in Zero-field-cooling $\left(\mathrm{M}_{Z F C}(\mathrm{~T})\right)$ and field-cooling $\left(\mathrm{M}_{F C}(\mathrm{~T})\right)$ are shown in Fig- 

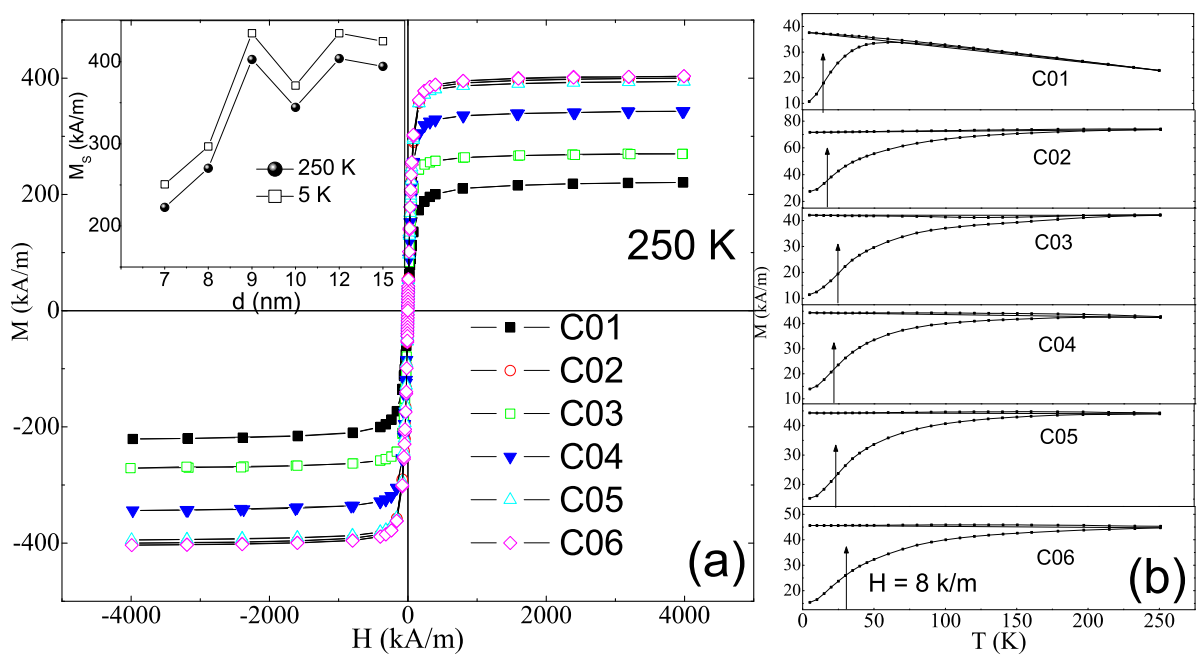

Fig. 4 (a) Magnetization loops of samples C01-06 measured at $250 \mathrm{~K}$ (the inset shows the dependence of MS with the mean diameter of the nanoparticles); (b) Zero-Field-Cooling and Field-Cooling curves measured with $\mathrm{H}=8 \mathrm{kA} / \mathrm{m}$. The arrows indicate the blocking temperature obtained from the maximum in the energy barrier distribution $((1 / \mathrm{T}) \mathrm{d}(\mathrm{MZFC}-\mathrm{MFC}) / \mathrm{dT} v s$. T) for each sample.

ure 4-b. By using the energy barrier distribution $\mathrm{f}(\mathrm{T})$ given by:

$$
f(T) \propto \frac{1}{T} \frac{d\left(M_{Z F C}-M_{F C}\right)}{d T}
$$

we obtain the blocking temperature $\mathrm{T}_{B}$ for all systems, given in Table 1 (and indicated by an arrow in the ZFC-FC curves in fig.4-b). By using the Néel model $\left(\tau=10^{-10} \exp \left(K_{e f f} V / k_{B} T_{B}\right)\right.$, where $\mathrm{V}$ is the particle volume, $\mathrm{K}_{e f f} \mathrm{~V}$ is the effective anisotropy barrier $\mathrm{E}_{a}$ and $\mathrm{k}_{B}$ is the Boltzmanns constant) and using the mean diameter from TEM analysis, we calculate the effective anisotropy constants $\mathrm{K}_{e f f}$, which are close to the expected one for the magnetocrystalline anisotropy constant for the bulk material $\left(\mathrm{K}_{e f f}=30-50 \mathrm{~kJ} / \mathrm{m}^{3}\right)(\mathrm{Kakol}$ et al (1989)).

From the measurements of the in-phase $\left(\chi^{\prime}\right)$ and out-of-phase $\left(\chi^{\prime \prime}\right)$ components of the ac susceptibility of our samples as a function of temperature and frequency, it is possible to determine the effective anisotropy constant $\mathrm{K}_{\text {eff }}(\mathrm{ac})$ and the characteristic relaxation time $\tau_{0}$ for our system. For that, we assume that the spin relaxation is thermally activated process, as proposed by the Néel model: $\mathrm{T}_{B}=K_{e f f} V / k_{B} \ln \left(1 / t_{0} f\right)$, and these values can be obtained from the linear fit of the values of $(1 / \mathrm{f}) v s$. $\mathrm{T}_{B}^{-1}$, where the blocking temperature $\mathrm{T}_{B}$ corresponds to the maximum in the out-of-phase component $\chi^{\prime \prime}(\mathrm{T}, \mathrm{f})$. The in-phase $\left(\chi^{\prime}\right)$ and out-of-phase $\left(\chi^{\prime \prime}\right)$ curves for samples C04-C06 are shown in the supplementary material, as representative of the systems. For samples CYY, a bimodal distribution of particles can be noticed with increasing number of re-growth steps. In figure 5, we present the plot of (1/f) $v s . \mathrm{T}_{B}^{-1}$ for samples C04, C05 and C06 with the corresponding linear fit. The values of $\mathrm{K}_{\text {eff }}(\mathrm{ac})$ values calculated are close to those ones obtained from the analysis of the $\mathrm{M}(\mathrm{T}) \mathrm{dc}$ measurements, and both are given in Table 1 together with the values obtained for $\tau_{0}$.

The magnetic parameters obtained for samples APXX, GEXX and CYY from magnetization and susceptibility measurements are given in Table 1. 


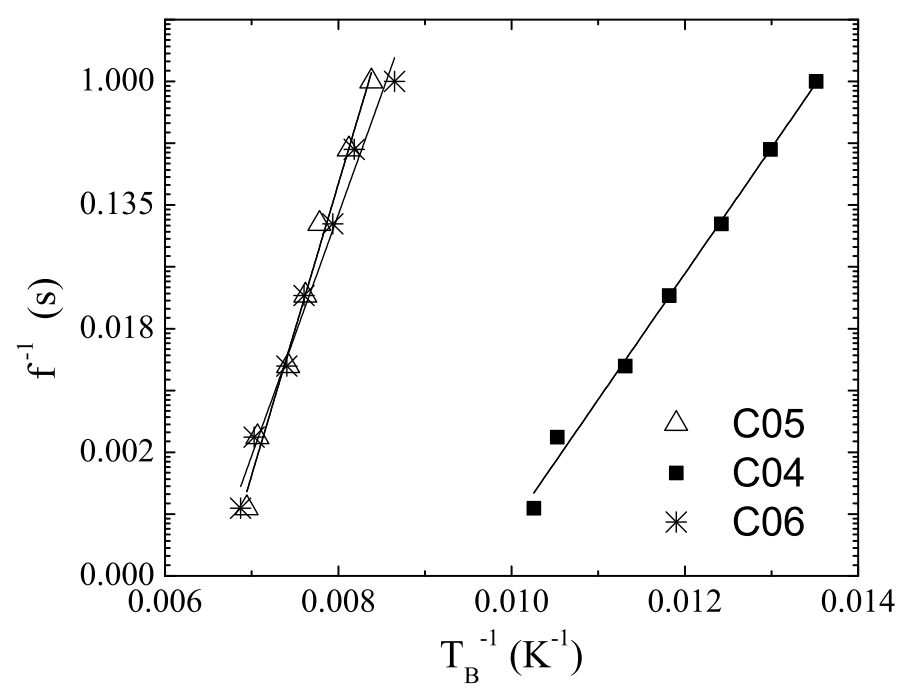

Fig. 5 Linear fit of (1/f) vs. $\mathrm{T}_{B}{ }^{-1}$ for samples $\mathrm{C} 04, \mathrm{C} 05$ and $\mathrm{C} 06$. The blocking temperature $\mathrm{T}_{B}$ corresponds to the maximum in the out-of-phase component $\left(\chi^{\prime \prime}\right)$ of the ac susceptibility for each frequency f. By using the Néel model, it is possible to estimate the values of $\mathrm{K}_{\text {eff }}$ and $\tau_{0}$.

\subsection{SPA Measurements}

For measuring the SPA of all samples, we applied an ac magnetic field ( $\mathrm{f}=250 \mathrm{kHz}, \mathrm{H}_{0}=$ $13 \mathrm{kA} / \mathrm{m}$ ) and extracted the time dependence of the temperature increase (Tvs. t) of the ferrofluid containing the NPs. The time dependence of the temperature for samples APXX, GEXX and CYY are presented in the supplementary material. As a reference for heat losses from the experimental setup, experiments with pure water were performed. The fastest response was observed for sample $\mathrm{C} 06$, which reached $\mathrm{T}>70^{\circ} \mathrm{C}$ in less than 5 min.. Experimentally, the SPA of a magnetic colloid constituted by a given mass of the nanoparticles $\mathrm{m}_{N P S}$ diluted in a mass of liquid carrier $\mathrm{m}_{L I Q}$ is evaluated by the Specific Power Absorption (SPA):

$$
S P A=\frac{P}{m_{N P s}}=\frac{\left.c_{L I Q} m_{L I Q}+c_{N P s} m_{N P S}\right)}{m_{N P S}} \frac{\Delta T}{\Delta t}
$$

where $\mathrm{c}_{L I Q}$ and $\mathrm{c}_{N P}$ are the specific heat capacities of the liquid carrier. Since the corresponding contributions for magnetite NPs in water at our standard concentration $(\sim 1 \% w t$. $)$ are $\mathrm{c}_{L I Q} \mathrm{~m}_{L I Q}=4.19 \mathrm{~J} / \mathrm{K}$ and $\mathrm{c}_{N P S} \mathrm{~m}_{N P}=6.7 \times 10^{-3} \mathrm{~J} / \mathrm{K}$, we can approximate $\mathrm{m}_{L I Q} \mathrm{c}_{L I Q}$ $+\mathrm{m}_{N P} \mathrm{c}_{N P} \sim \mathrm{m}_{L I Q} \mathrm{c}_{L I Q}$ and write eq. 2 as:

$$
S P A=\frac{c_{L I Q} m_{L I Q}}{m_{N P S}} \frac{\Delta T}{\Delta t}
$$

Therefore, we can obtain SPA from derivation of the curve T vs. t for all samples. For accurate estimation of SPA values of magnetic colloids, experiments should be done in adiabatic conditions (Natividad et al (2009)). To avoid heat losses bu exchange with the sample environment (sample holder walls, irradiation, etc) we have used the criterion of the criterion of the maximum of the derivative $d T / d t$ to determine SPA. As the maximum increase always occurs within the first few seconds of the experiment, the adiabatic character 


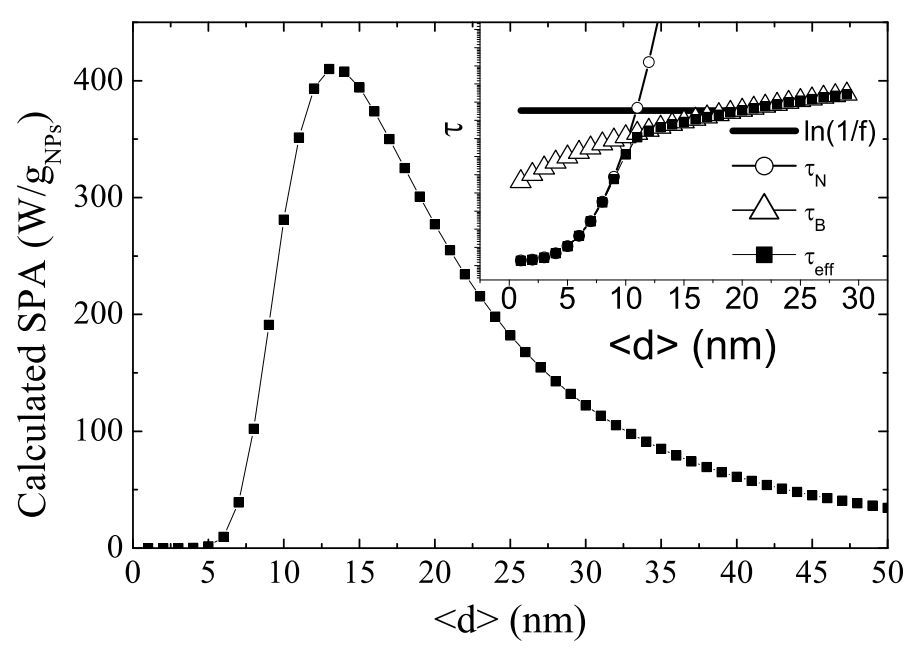

Fig. 6 Calculated SPA vs. d curves of nanoparticles dispersed in toluene for an alternating magnetic field with $\mathrm{H}_{0}=13 \mathrm{kA} / \mathrm{m}$ and $\mathrm{f}=250 \mathrm{kHz}$, and assuming the magnetic parameters characteristics of $\mathrm{Fe}_{3} \mathrm{O}_{4}$ nanoparticles with size dispersion of $\sigma=0.20: \mathrm{K}_{e f f}=3 \mathrm{~kJ} / \mathrm{m}^{3}, \mathrm{M}_{S}=450 \mathrm{kA} / \mathrm{m}$ and $\tau_{0}=10^{-10} \mathrm{~s}$. Hydrodynamic radius was considered as $\left\langle\mathrm{d}_{H}\right\rangle=\langle\mathrm{d}\rangle+3 \mathrm{~nm}$. Inset presents the size dependence of $\tau_{N}, \tau_{B}$ and $\tau_{e f f}$.

Table 1 Results of TEM, Magnetic and SPA measurements for samples APXX, GEXX and CYY.

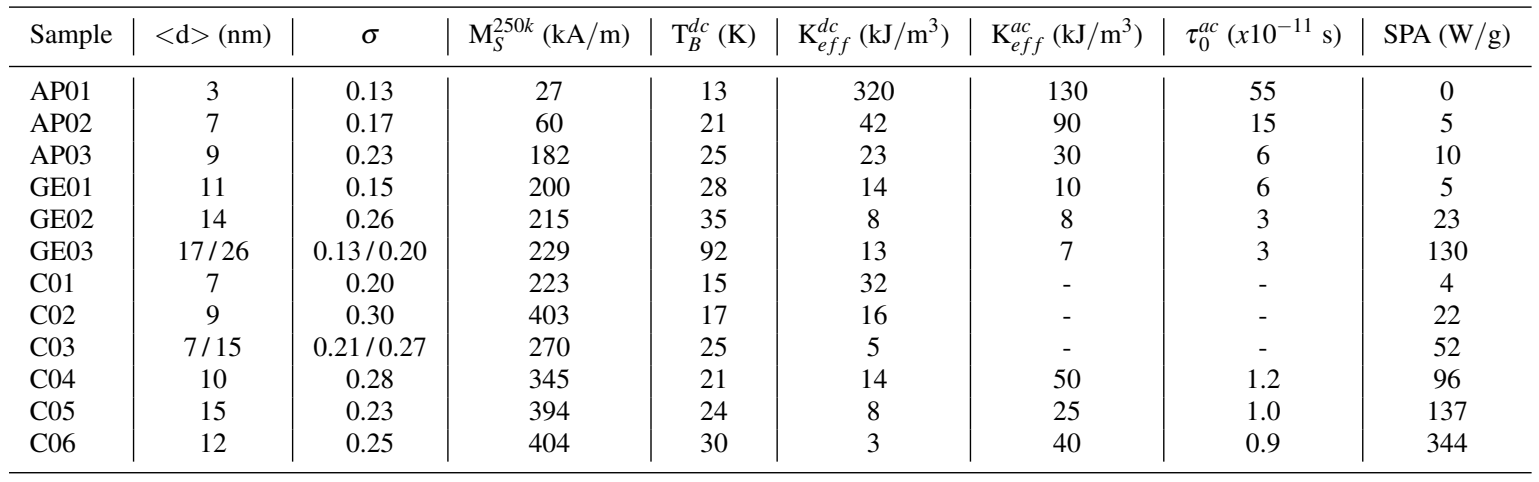

of the measurement is granted. Additionally, this criterion of maximum derivative can be taken as a rule for comparing data from different authors, irrespective of the exact shape of the $\mathrm{T}(\mathrm{t})$ curves, which often depends on the experimental details. All SPA values are given in Table 1.

\section{Discussion}

In order to estimate the influence of Néel relaxation in the SPA observed for our systems, we extrapolated the blocking temperature of sample CYY for the SPA experiment conditions ( $\mathrm{f}$ $=250 \mathrm{kHz}$ and $\mathrm{H}_{0}=13 \mathrm{kA} / \mathrm{m}$ ) by using the Néel Model:

$$
\tau_{N}=\tau_{0} \exp \left(\frac{K_{e f f} V\left(1-H M_{S} / 2 K\right)^{2}}{k_{B} T}\right)
$$


where $\tau_{N}$ is the Néel fluctuation time, $\mathrm{M}_{S}$ is the magnetization saturation, $\mathrm{V}$ is the particle mean volume, $\tau_{0}$ is the characteristic fluctuation time $\left(\sim 10^{-9}-10^{-10} \mathrm{~s}\right), \mathrm{K}_{\text {eff }}$ is the effective anisotropy constant and $\mathrm{k}_{B}$ is the Boltzmanns constant. We calculated for the sample with $\mathrm{d}=17 \mathrm{~nm}$ a $\mathrm{T}_{B} \sim 380 \mathrm{~K}$, and for the other samples, excepting those with bimodal size distribution, we obtained $\mathrm{T}_{B}$ close or lower than room temperature. So, almost all samples will be in the superparamagnetic regime in the conditions of our SPA measurements. Hydrodynamic radius $\mathrm{d}_{h}$ where measured for these samples by light scattering, being around 12-30 $\mathrm{nm}$. In fact, we can consider that $\mathrm{d}_{h}$ is approximately the mean diameter plus the organic layer of oleic acid in the surface (around 1-3 $\mathrm{nm}$ ). The Brown relaxation time can be obtained by $\tau_{B}=3 \eta \mathrm{V}_{h} / \mathrm{k}_{B} \mathrm{~T}$, where $\eta$ is the viscosity of the liquid and $\mathrm{V}_{h}$ is the hydrodynamic volume of the particles. We obtain that the $\tau_{B}$ of all samples is lower than the characteristic time of our SPA experiment (1/f) for all mean diameters involved, even when considering the presence of the organic layer with thickness estimated of 1-3 $\mathrm{nm}$. So, the Brown relaxation mechanism is expected to have strong influence in the heating generations of all samples. In this way, we could not expect heating generation as consequence of hysteresis area as expected for blocked nanoparticles with larger hydrodynamic radius; in this case, Brown relaxation will be dominant.

According to Rosensweig (2002), both Brown and Néel relaxation processes occurs in parallel and the effective relaxation time is given by:

$$
\tau_{e f f}^{-1}=\tau_{B}^{-1}+\tau_{N}^{-1}
$$

The out-of-phase component $\left(\chi^{\prime \prime}\right)$ the susceptibility is given by:

$$
\chi^{\prime \prime}=\frac{2 \pi \chi_{0} f \tau_{e f f}}{1+\left(2 \pi \tau_{e f f} f\right)^{2}}
$$

where $\chi_{0}$ is the static susceptibility of the system. According to eq. $6, \chi^{\prime \prime}$ presents a maximum for $2 \pi \mathrm{f}=\tau_{e f f}^{-1}$, consequently SPA also presents a maximum when this condition is attempted. In this way, the SPA of the system can be calculated by:

$$
S P A=\mu_{0} H_{0}^{2} f \pi \chi^{\prime \prime}
$$

In real colloids the constituent nanoparticles have a distribution of particle sizes around a mean value $<\mathrm{d}>$. The statistical mean value and standard deviation of this size distribution have a strong effect on the resulting SPA values, and must therefore be taken into account by the convolution of the SPA and distribution function:

$$
S P A(<d>)={ }_{0} \int^{\infty} S P A(x) P(x) d x
$$

where $\mathrm{P}(\mathrm{x})$ is the distribution function of the particles. For the usual size distributions found in most synthetic MNPs, the effect of the distribution function $\mathrm{P}(\mathrm{x})$ in Eq. 8 is to reduce the maximum, while broadening the peak profile of the $\operatorname{SPA}\left(i_{i}\right)$ curve.

We reinforce that the application of the model presented above for the heat generation requires one of these conditions: a) Superparamagnetic regime at room temperature and 250 $\mathrm{kHz}$, and/or b) a small relative small hydrodynamic diameter, without the formation of agglomeration. These two conditions make that $\tau_{B}$ and $\tau_{N}$ values are smaller than $1 / 2 \pi f$. The ac susceptibility and magnetization measurements together with the determination of $\mathrm{V}_{h}$ show that our samples accomplish at least one of these necessary characteristics. Magnetization measurement as function of applied field of sample GE03 exhibits a small hysteresis at room temperature, which can be interpreted that hysteresis losses can be important for the 
heating generation of this sample. However, it is important to note that the magnetic measurements are performed in nanoparticles that are immobilized, in contrast to the situation of the SPA experiments where the MNPs are suspended in a liquid carrier and can thus relax through Brown mechanism.

Therefore, assuming that the Néel and Brown relaxation mechanisms are independently, the SPA values can be calculated by Eq. 8 with using the structural and magnetic parameters presented in Table 1, together with the hydrodynamic diameter.

Fig. 6 presents the calculated dependence of SPA with the diameter of nanoparticles dispersed in toluene for an alternating magnetic field with $\mathrm{H}_{0}=13 \mathrm{kA} / \mathrm{m}$ and $\mathrm{f}=250 \mathrm{kHz}$, and assuming the magnetic parameters characteristic of ferrite nanoparticles with size dispersion of $\sigma=0.20, \mathrm{~K}_{e f f}=50 \mathrm{~kJ} / \mathrm{m}^{3}, \mathrm{M}_{S}=512 \mathrm{kA} / \mathrm{m}$ and $\tau_{0}=10^{-10} \mathrm{~s}$. We also consider that a monolayer of oleic acid covers the nanoparticles, as in our samples, with characteristic thickness of $3 \mathrm{~nm}$. Therefore, hydrodynamic radius was considered as $\left\langle d_{h}\right\rangle=\langle\mathrm{d}\rangle+6$ $n m$. Inset of fig. 6 presents the size dependence of $\tau_{N}, \tau_{B}$ and $\tau_{e f f}$, together with the values of $1 / \mathrm{f}$, which marks the limit where the hysteresis losses contributes to the heating generation.

As observed in fig. 6, there is a strong dependence of the SPA with the particle mean diameter $\langle\mathrm{d}\rangle$, with a maximum of SPA $(\sim 400 \mathrm{~W} / \mathrm{g})$ for $\mathrm{d}=13 \mathrm{~nm}$. Several works (GonzalezFernandez et al (2009), Gonzales-Weimuller et al (2009), Levy et al (2008) and Vaishnava et al (2007)) have previously presented by experimental and numerical calculations the strong dependence of the SPA on particle volume, which is due to the volume dependence of both Néel and Brown relaxation times. The key parameters to the Brown relaxation time is the hydrodynamic radius of the particle and the viscosity of the fluid, while for the Néel relaxation of single-domain nanoparticles, the relevant parameter is the product $\mathrm{E}_{a}=\mathrm{K}_{e f f} \mathrm{~V}$, where $\mathrm{K}_{\text {eff }}$ is the effective anisotropy constant and $\mathrm{V}$ is the particle volume. Therefore, these parameters are determinant for the SPA values observed for our samples.

Fig. 7 present the measured value of SPA for samples APXX, GEXX, and fig. 6-b presents the equivalent curve for samples CYY, with maximum of $344 \mathrm{~W} / \mathrm{g}$ at $\mathrm{d}=12 \mathrm{~nm}$ for sample C06. In the same figure, we also present the calculated SPA values (eq.8) with considering the Néel and Brown relaxations and using the morphological and magnetic parameter given in Table 1. We observe the good agreement of our experimental, despite the bimodal diameter distribution, with the presence of very small nanoparticles $(<5 \mathrm{~nm})$ observed in all samples grown by the seeds procedure, are not take into account, which would lead to a reduction in the calculated SPA values. It is more evident for samples GEXX, where the larger dispersion leads to a mismatch between and experimental values. Concerning the high value of SPA observed for sample GE03, it is due to the $17 \mathrm{~nm}$ particles, since our calculations indicate a lower SPA for the nanoparticles with $\mathrm{d} \sim 20 \mathrm{~nm}$ in this sample. Notice that Néel relaxation time has an exponential with $\mathrm{V}$, while Brown relaxation time presents a linear one. Thus, the Néel relaxation will dominate the heating generation for smaller diameters (d $<11 \mathrm{~nm}$ for $\mathrm{f}=250 \mathrm{kHz}$ ), while Brown relaxation dominates for larger ones $(\mathrm{d}=20 \mathrm{~nm}$ for $\mathrm{f}=250 \mathrm{kHz}$ ). The Néel and Brown relaxation times have the same magnitude for a narrow range of diameters $(11 \mathrm{~nm}<\mathrm{d}<13 \mathrm{~nm})$, where both relaxations contribute significantly for the heating generation. Brown relaxation should be considered in the hysteresis area of the blocked nanoparticles, which has no easy analytical solution Carrey et al (2011). For larger nanoparticles, the heating generation will be determined by the hysteresis are of the nanoparticles, and the optimization of the nanoparticle parameters for hyperthermia should be obtained in another way (Carrey et al (2011), Usov (2010) and Seung-Hyun et al (2012)). An additional drawback in comparing SPA values from different authors is the field- and frequency dependence of this parameter. A single expression including the effects of mag- 


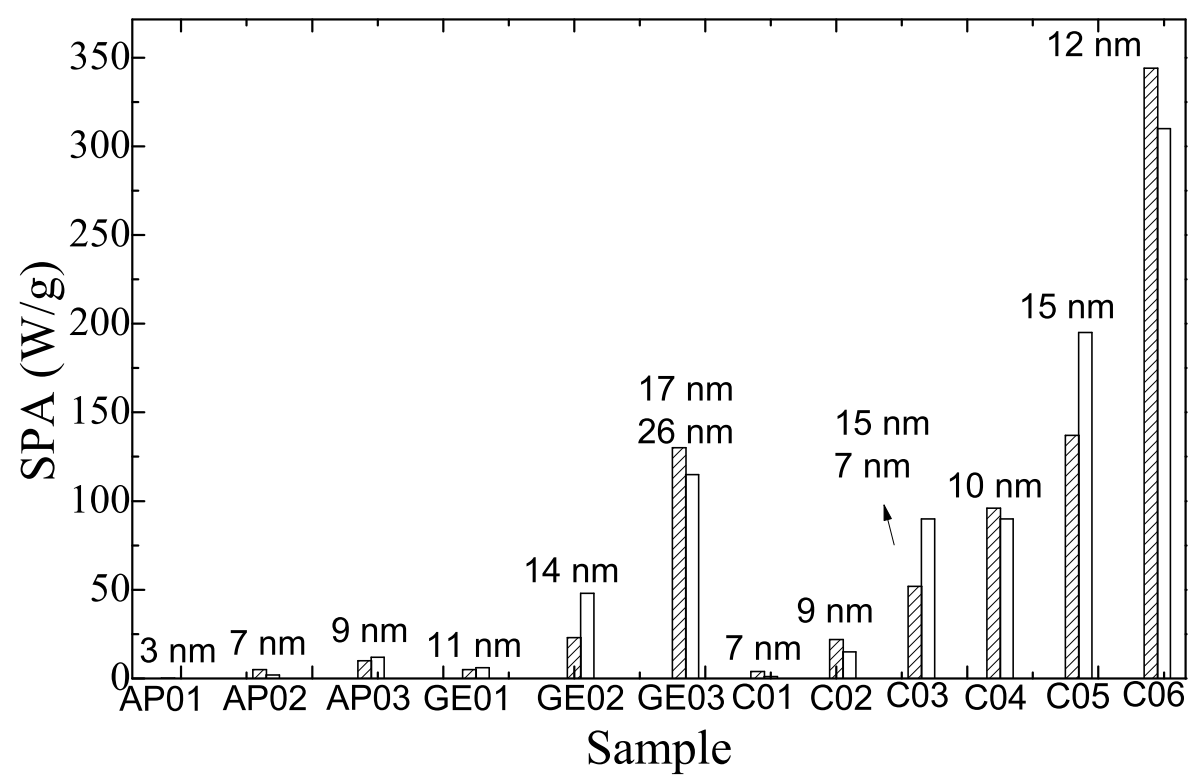

Fig. 7 Comparison between experimental SPA values of samples APXX and CYY with calculated ones (eq. 8) with assuming the Néel and Brown relaxations and the morphological and magnetic parameters of each sample given in Table 1

netic field and frequency of the actual measurement is needed if different experiments are to be compared. Since SPA values are dependent on the amplitude and frequency of the magnetic field applied in each experiment, comparison between heating performance of different MNPs through this parameter is seldom illustrative. To solve this difficulty, Kallumadil et al (2009) have discussed the use of a system-independent parameter, called intrinsic loss power (ILP), which is defined as $I L P=S P A / f H^{2}$. The ILP value was though as independent of the field- and frequency, for $\mathrm{f}$ values on the $\mathrm{kHz}$ range and $\mathrm{H}<<H_{C}$, where $H_{C}$ is the coercivity of the magnetic material. Usual values found in the literature range between 0 and 4 $n H . m^{2} . \mathrm{kg}^{-1}$, the highest reported so far being $3.1 \mathrm{nH} . \mathrm{m}^{2} . \mathrm{kg}^{-1}$ for commercial (Micromod Nanomag-D-Spio) samples with average crystallite size of $11.8(0.56) \mathrm{nm}$. When converted into ILP we obtained values between 0 (for sample AP01) up to $8.1 \mathrm{nH} . \mathrm{m}^{2} . \mathrm{kg}^{-1}$ (for sample C06). We remark that the concept of the ILP is more accurate if it is assumed as an estimation of the capability of the system to absorb the power of the ac magnetic field (which is proportional to $f . H^{2}$ ).// SPA is not only dependent on the particle size, but also depends on the formation of agglomerates of the magnetic interparticle interaction, since it leads to the increment of the blocking temperature and to the strongly increment of the hydrodynamic radius, making no valid the heating generation as originated by Néel and Brown relaxations, being dependent of the hysteresis losses in a blocked regime Carrey et al (2011). In this case the parameters to be optimizes for maximum SPA values will be different, demonstrating the important role of colloidal stability of the ferrofluid in the final power absorption efficiency. This observation opens the question of how chemical changes into physiological medium ( $\mathrm{pH}$, salinity, etc.) will affect the SPA of a given colloid for biomedical applications. 


\section{Summary}

Summarizing, experimental study and numerical calculations of SPA as a function of particle size of $\mathrm{Fe}_{3} \mathrm{O}_{4}$ nanoparticles show that morphological (the average size and size distribution) and magnetic (anisotropy constant and relaxation time) characteristics of the nanoparticles constituting a heating agent are central parameters for the design of efficient heating agents. Numerical calculations considering the heating generation due to the Néel and Brown relaxations are in good agreement with experimental data. Looking at the mechanisms of heating generation, our numerical calculations indicates that for our experimental conditions ( $\mathrm{f}=250 \mathrm{kHz}$ and $\mathrm{H}_{0}=13 \mathrm{kA} / \mathrm{m}$ ) the Néel relaxation is predominant for $\mathrm{d}<$ $11 \mathrm{~nm}$, Brown and Néel relaxations compete for $11 \mathrm{~nm}<\mathrm{d}<13 \mathrm{~nm}$ and Brown relaxation dominates for $\mathrm{d}=20 \mathrm{~nm}$. SPA value as large as $344 \mathrm{~W} / \mathrm{g}$ was obtained for a sample containing nanoparticles with average size of $12 \mathrm{~nm}$ in an ac magnetic field with $\mathrm{H}_{0}=13 \mathrm{kA} / \mathrm{m}$ and $\mathrm{f}=250 \mathrm{kHz}$. Based in our results, it is possible to design nanoparticles with morphological and magnetic properties that optimize the heating generation, in accordance with the frequency and amplitude of the ac magnetic field, by the Néel and Brown relaxations.

\section{Declaration of Interest}

The authors declare that they do not have any affiliations that would lead to a conflict of interest.

Acknowledgements This work was supported partially from Diputación General de Aragon (DGA) and Ministerio de Economia y Competitividad (MINECO, project MAT2010-19326), Spain. Partial support from the Brazilian agency FAPESP is also acknowledged. E. Lima Jr. acknowledges financial support from the FAPESP through a postdoctoral fellowship.

\section{References}

KIM, B. H. , Lee N., Kim H., An K., Park Y. I., Choi Y., Shin K., Lee Y., Kwon S. G., Na H. B. 2011. LargeScale Synthesis of Uniform and Extremely Small-Sized Iron Oxide Nanoparticles for High-Resolution T-1 Magnetic Resonance Imaging Contrast Agents. J Am Chem Soc 133:(32) 12624-12631.

Maier-Hauff K., lrich F., Nestler D., Niehoff H., Wust P., Thiesen B., Orawa H., Budach V., Jordan A. 2011 Efficacy and safety of intratumoral thermotherapy using magnetic iron-oxide nanoparticles combined with external beam radiotherapy on patients with recurrent glioblastoma multiforme. J Neuro-Oncol $103:(2) 317-324$.

van Landeghem F. K. H., Maier-Hauff K., Jordan A., Hoffmann K. T., Gneveckow U., Scholz R., Thiesen B., Bruck W., von Deimling A. 2009 Post-mortem studies in glioblastoma patients treated with thermotherapy using magnetic nanoparticles.Biomaterials 30:(1) 52-57.

Kakol Z., Pribble R. N., Honig J. M. 1989 Magnetocrystalline anisotropy of $\mathrm{Fe}_{3(1-d)} \mathrm{O}_{4}, 0=d<0.01$. Solid Stat Commun 69:(7) 793-796.

Johannsen M., Thiesen B., Wust P., Jordan A. 2010 Magnetic nanoparticle hyperthermia for prostate cancer. Int J Hyperther 26:(8) 790-795.

Thiesen B., Jordan A. 2008 Clinical applications of magnetic nanoparticles for hyperthermia. Int J Hyperther 24:(6) 467-474.

Alexander H. R. 2003 Hyperthermia and its modern use in cancer treatment. Cancer 98:(2) 219-221.

Goya G. F., Grazu V., Ibarra M. R. 2008 Magnetic nanoparticles for cancer therapy. Curr Nanosci 4:(1) 1-16.

Tanaka K., Narita A., Kitamura N., Uchiyama W., Morita M., Inubushi T., Chujo Y. 2010 Preparation for Highly Sensitive MRI Contrast Agents Using Core/Shell Type Nanoparticles Consisting of Multiple SPIO Cores with Thin Silica Coating. Langmuir 26:(14) 11759-11762.

Alphandery E., Faure S., Raison L., Duguet E., Howse P. A., Bazylinski D. A. 2011 Heat Production by Bacterial Magnetosomes Exposed to an Oscillating Magnetic Field. J Phys Chem C 115:(1) 18-22. 
Hergt R., Hiergeist R., Zeisberger M., Schuler D., Heyen U., Hilger I., Kaiser W. A. 2005 Magnetic properties of bacterial magnetosomes as potential diagnostic and therapeutic tools J Magn Magn Mater 293:(1) 8086.

Goya G. F., Lima Jr. E., Arelaro A. D., Torres T. E., Rechenberg H. R., Rossi L., Marquina C., Ibarra M. R. 2008 Magnetic Hyperthermia With $\mathrm{Fe}_{3} \mathrm{O}_{4}$ Nanoparticles: The Influence of Particle Size on Energy Absorption. IEEE T Magn 44:(11) 4444-4447.

Goya G. F., Fernandez-Pacheco R., Arruebo M., Cassinelli N., Ibarra M. R. 2007 Brownian rotational relaxation and power absorption in magnetite nanoparticles J Magn Magn Mater 316:(2) 132-135.

Carrey J., Mehdaoui B., Respaud M. 2011 Simple models for dynamic hysteresis loop calculations of magnetic single-domain nanoparticles: Application to magnetic hyperthermia optimization. J Appl Phys 109:(3) 083921.

Rosensweig R. E. 2002 Heating magnetic fluid with alternating magnetic field. J Magn Magn Mater 252:(13)370-374.

Sun S. H., Zeng H., Robinson D. B., Raoux S., Rice P. M., Wang S. X., Li G. X. 2004 Monodisperse MFe2O4 (M = Fe, Co, Mn) nanoparticles J Am Chem Soc 126:(1) 273-279.

Sun S. H., Zeng H. 2002 Size-controlled synthesis of magnetite nanoparticles. J Am Chem Soc 124:(28) 82048205 .

Torres T. E., Roca A. G., Morales M. P., Ibarra A., Marquina C., Ibarra M. R., Goya G. F. 2010 Magnetic properties and energy absorption of $\mathrm{CoFe}_{2} \mathrm{O}_{4}$ nanoparticles for magnetic hyperthermia J Phys Conf Ser 200:(7) 072101.

Natividad E., Castro M., Mediano A. 2009 Adiabatic vs. non-adiabatic determination of specific absorption rate of ferrofluids J Magn Magn Mater 321:(10) 1497-1500.

Gonzalez-Fernandez M. A., Torres T. E., Andres-Verges M., Costo R., de la Presa P., Serna C. J., Morales M. R., Marquina C., Ibarra M. R., Goya G. F. 2009 Magnetic nanoparticles for power absorption: Optimizing size, shape and magnetic properties J Solid State Chem 182:(10) 2779-2784.

Gonzales-Weimuller M., Zeisberger M., Krishnan K. M. 2009 Size-dependant heating rates of iron oxide nanoparticles for magnetic fluid hyperthermia J Magn Magn Mater 321:(13) 1947-1950.

Levy M., Wilhelm C., Siaugue J. M., Horner O., Bacri J. C., Gazeau F. 2008 Magnetically induced hyperthermia: size-dependent heating power of $\gamma$-Fe(2)O(3) nanoparticles J Phys-Condens Mat 20:(20) 204133.

Vaishnava P. P., Tackett R., Dixit A., Sudakar C., Naik R., Lawes G. 2007 Magnetic relaxation and dissipative heating in ferrofluids J Appl Phys 102:(6) 063914.

Usov N. A. 2010 Low frequency hysteresis loops of superparamagnetic nanoparticles with uniaxial anisotropy J Appl Phys 107:(12) 123909.

Seung-hyun Noh, Wonjun Na, Jung-tak Jang, Jae-Hyun Lee, Eun Jung Lee, Seung Ho Moon 2012 Nanoscale Magnetism Control via Surface and Exchange Anisotropy for Optimized Ferrimagnetic Hysteresis Nano Lett dx.doi.org/10.1021/n1301499u.

Kallumadil M., Tada M., Nakagawa T., Abe M., Southern P., Pankhurst Q. A. 2009 Suitability of commercial colloids for magnetic hyperthermia J Magn Magn Mater 321:(21) 3650-3651.

Vargas J. M., Zysler R. D. 2005 Tailoring the size in colloidal iron-oxide magnetic nanoparticles. Nanotechnology 16:(9) 1474-1476. 